\title{
BIOLOGÍA REPRODUCTIVA DEL MOLUSCO BIVALVO Mesodesma donacium LAMARCK, 1819
}

Roberto Castellanos Cabrera ${ }^{\prime}$ Manuela Cosavalente Sánchez ${ }^{2}$

\author{
RES UMEN
}

El estudio reproductivo de Mesodesma donacium LAMARCK, 1818 de la localidad de Pozo Lisas, ubicada en el puerto de llo-Moquegua, ha sido realizado en el periodo comprendido entre abril 1992 y marzo 1993.

El material biológico estuvo constituido por 1800 ejemplares de Mesodesma donacium que han sido obtenidos mensualmente del banco natural ubicado en la playa arenosa de Pozo Lisas. La proporción sexual fué de 1:1 y la talla máxima encontrada fue de $92.5 \mathrm{~mm}$. El ciclo reproductivo comprende tres estados: inmaduro (marzo-julio), maduro (agosto-octubre) y en desove (todo el año, con mayor frecuencia en noviembre y febrero). La primera madurez sexual se manifiesta a los $60.0 \mathrm{~mm}$. de longitud total, siendo el promedio de individuos maduros de $77.2 \mathrm{~mm}$.

\section{A B S T R A C T}

The reproductive study of Mesodesma donacium LAMARCK 1818 in the locality of Pozo Lisas, situated in the port of Ilo-Moquegua, has been carried out in three periods between April 1992 and march 1993.

The biological material was constituted by 1800 examples of Mesodesma donacium which were obtained on a monthly basis in the natural bank located at the sandy beach of Pozo Lisas. The sexual proportion was $1: 1$ and the maximum size found was $92.5 \mathrm{~mm}$. The reproductive cycle has 3 states : immature (March-July), mature (August-October) and spawning (all year with greater frequency in November and Febraury). The first sexual maturity appears at a total length of $60.0 \mathrm{~mm}$, the average site of mature specimens being $77.2 \mathrm{~mm}$. 


\section{INTRODUCCIÓN}

Mesodesma donacium Lamarck, 1818 es una de las especies más comunes de las playas arenosas del litoral de nuestro país, y en la zona sur, se encuentra en los litorales arenosos de Tacna, Moquegua y Arequipa, distribuida desde la Concordia, límite con el vecino país de Chile hasta la playa arenosa de Vila Vila (Tacna). Más al norte se encuentra en las playas arenosas de Pozo Lisas, cerca al puerto de llo, Moquegua. Schweigger (1964) menciona su frecuencia desde Trujillo, en el Perú, hasta la zona central de Chile. Osorio y Bahamonde (1968) registran su distribución desde la bahía de Sechura en el Perú hasta Valparaíso en Chile.

Entre las informaciones existentes sobre el aspecto reproductivo de Mesodesma donacium se cuentan los trabajos de López (1986) realizado en Chile y de Salgado e Ishiyama (1979) en Camaná - Perú, Castellanos \& Col. $(1980,1981,1982,1983)$. Trabajos en especies similares han sido realizados por Christiansen (1981) en Mesodesma mactroides de Argentina y Redfearn (1974) en Paphies (Mesodesma) ventricosa de Nueva Zelandia.

Se ha realizado el estudio reproductivo de Mesodesma donacium de la localidad de Pozo Lisas - Moquegua, incidiendo principalmente en el estudio de la primera madurez sexual, periodo de desove y el ciclo reproductivo, lo que permitirá reconsiderar el tamaño mínimo de extracción y el periodo de veda para la zona de Moquegua, a fin de proteger a este recurso de la depredación y la sobreexplotación a que viene siendo sometido actualmente.

\section{MATERIAL Y MÉTODOS}

El material biológico estuvo constituido por 1800 ejemplares de Mesodesma donacium (Lamarck, 1818), obtenidos mensualmente del banco natural ubicado en la playa arenosa de Pozo Lisas, puerto de Ilo - Moquegua. Los muestreos poblacionales se realizaron mensualmente a partir del mes de abril de 1992 hasta marzo de 1993, a razón de 150 individuos por muestreo al azar, haciendo un total de 1800 ejemplares. De cada muestreo poblacional se han tomado las más representativas para los estudios histológicos gonadales, consistiendo en un número exacto de 50 ejemplares por cada mes de muestreo. El número total de ejemplares analizados fue de 600 , obteniéndose además los datos biométricos referentes a longitud total $(\mathrm{mm})$, peso total $(\mathrm{g})$ y peso comestible (g). El sexo y el estado sexual ha sido determinado empleando el tratamiento histológico gonadal. Para la obtención de la muestra gonadal se ha procedido mediante el muestreo estratificado aleatorio, agrupando clases de longitudes de $2 \mathrm{~mm}$ de intervalo.
Las muestras seleccionadas para los estudios reproductivos han sido conservadas en formalina al $10 \%$, utilizando agua de mar, luego en el laboratorio se procedió a obtener la muestra gonadal consistente en una sección transversal media del pie, siendo posteriormente fijada para seguir con el tratamiento histológico correspondiente, realizando los cortes histológicos, coloración y montaje siguiendo la metodología establecida por Difiore (1969).

El análisis histológico de las muestras gonadales se ha realizado empleando la escala de madurez sexual de Chistiansen (1981), obtenida durante el estudio del ciclo reproductivo de Mesodesma mactroides, habiendo sido esta escala también utilizada por Salgado \& Ishiyama (1979) para el estudio del ciclo reproductivo de Mesodesma donacium de la zona de Camaná (Arequipa), para efectos de una mejor interpretación se ha procedido, al igual que Christiansen (1981), a modificar los estadios sexuales.

\section{RESULTADOS}

\subsection{PROPORCIÓN SEXUAL}

Los resultados obtenidos a través del tratamiento estadístico de los datos de frecuencia por sexos, durante los meses comprendidos entre los meses de abril de 1992 hasta marzo de 1993 pueden observarse en la Tabla $N^{\circ} 1$. Ella contiene la proporción sexual mensual de la macha Mesodesma donacium, dándose dicha proporción en forma porcentual Al final de las proporciones mensuales consideramos la proporción sexual anual. Para la determinación de la proporción sexual de la macha se ha planteado la hipótesis siguiente :

$$
\begin{aligned}
& \text { Ho: № de machos }=\text { № de hembras } \\
& \mathrm{Ha}: \text { № de machos }=\text { № de hembras }
\end{aligned}
$$

\subsection{CICLO REPRODUCTIVO DE M. DONACIUM L.}

El ciclo reproductivo de $M$. donacium en el presente estudio (Fig.1) se ha determinado siguiendo la clasificación de los estados de desarrollo gonadal, expuestos en el capitulo de material y métodos; considerándose los sexos combinados. Estos estadios fueron:

\subsubsection{Inmaduro}

Se presentó con mayor incidencia durante los meses comprendidos entre marzo y julio, mostrando un pico máximo en el mes de abril (Tabla II; Fig.1), representando el $18.5 \%$ de la muestra total analizada. Las microfotografías A muestran las caracteristicas histológicas de este estado, tanto en ejemplares machos como en hembras, correspondiendo dichas caracteristicas a las consideradas en la bibliografía consultada. 
TABLA $N^{\circ} I$

PROPORCIÓN SEXUAL MENSUAL DE M.

donacium $L$.

POZO LISAS (PTO. DE ILO-MOQUEGUA) 1992-93

\begin{tabular}{|l|rc|rc|}
\hline MESES & \multicolumn{2}{|c|}{ M A C H O S } & \multicolumn{2}{|c|}{ H E M B R A S } \\
& $n_{1}$ & $\%$ & $n_{2}$ & $\%$ \\
\hline ABRIL/1992 & 30 & 60.0 & 20 & 40.0 \\
MAYO & 23 & 46.0 & 27 & 54.0 \\
JUNIO & 25 & 50.0 & 25 & 50.0 \\
JULIO & 31 & 62.0 & 19 & 38.0 \\
AGOSTO & 27 & 54.0 & 23 & 46.0 \\
SETIEMBRE & 24 & 48.0 & 26 & 52.0 \\
OCTUBRE & 22 & 44.0 & 28 & 56.0 \\
NOVIEMBRE & 33 & 66.0 & 17 & 34.0 \\
DICIEMBRE & 31 & 62.0 & 19 & 38.0 \\
ENERO/1993 & 33 & 66.0 & 17 & 34.0 \\
FEBRERO & 30 & 60.0 & 20 & 40.0 \\
MARZO & 24 & 48.0 & 26 & 52.0 \\
TOTAL & 333 & 55.5 & 267 & 44.5 \\
\hline
\end{tabular}

TABLA $N^{\circ} 2$

CICLO REPRODUCTIVO DE LA MACHA M. donacium LAMARCK, 1818

\begin{tabular}{|c|c|c|c|c|c|c|}
\hline \multirow[t]{2}{*}{ MESES } & \multicolumn{2}{|c|}{ INMADURO } & \multicolumn{2}{|c|}{ MADURO } & \multicolumn{2}{|c|}{ EN DESOVE } \\
\hline & $n_{1}$ & $\%$ & $\mathrm{n}_{2}$ & $\%$ & $\mathrm{n}_{3}$ & $\%$ \\
\hline Abril 1992 & 28 & 56.0 & 6 & 12.0 & 16 & 32.0 \\
\hline Mayo & 24 & 48.0 & 6 & 12.0 & 20 & 40.0 \\
\hline Junio & 16 & 32.0 & 8 & 16.0 & 26 & 52.0 \\
\hline Julio & 12 & 24.0 & 9 & 18.0 & 29 & 58.0 \\
\hline Agosto & 4 & 8.0 & 26 & 52.0 & 20 & 40.0 \\
\hline Setiembre & 2 & 4.0 & 43 & 86.0 & 5 & 10.0 \\
\hline Octubre & 1 & 2.0 & 45 & 90.0 & 4 & 8.0 \\
\hline Noviembre & 2 & 4.0 & 4 & 8.0 & 44 & 88.0 \\
\hline Diciembre & 1 & 2.0 & 3 & 6.0 & 46 & 92.0 \\
\hline Enero 1993 & 2 & 4.0 & 4 & 8.0 & 44 & 88.0 \\
\hline Febrero & 3 & 6.0 & 5 & 10.0 & 42 & 84.0 \\
\hline Marzo & 16 & 32.0 & 6 & 12.0 & 28 & 56.0 \\
\hline TOTAL & 111 & & 165 & & 32 & \\
\hline Porcentaje & 18.5 & & 27.5 & $\%$ & 54.0 & \\
\hline
\end{tabular}

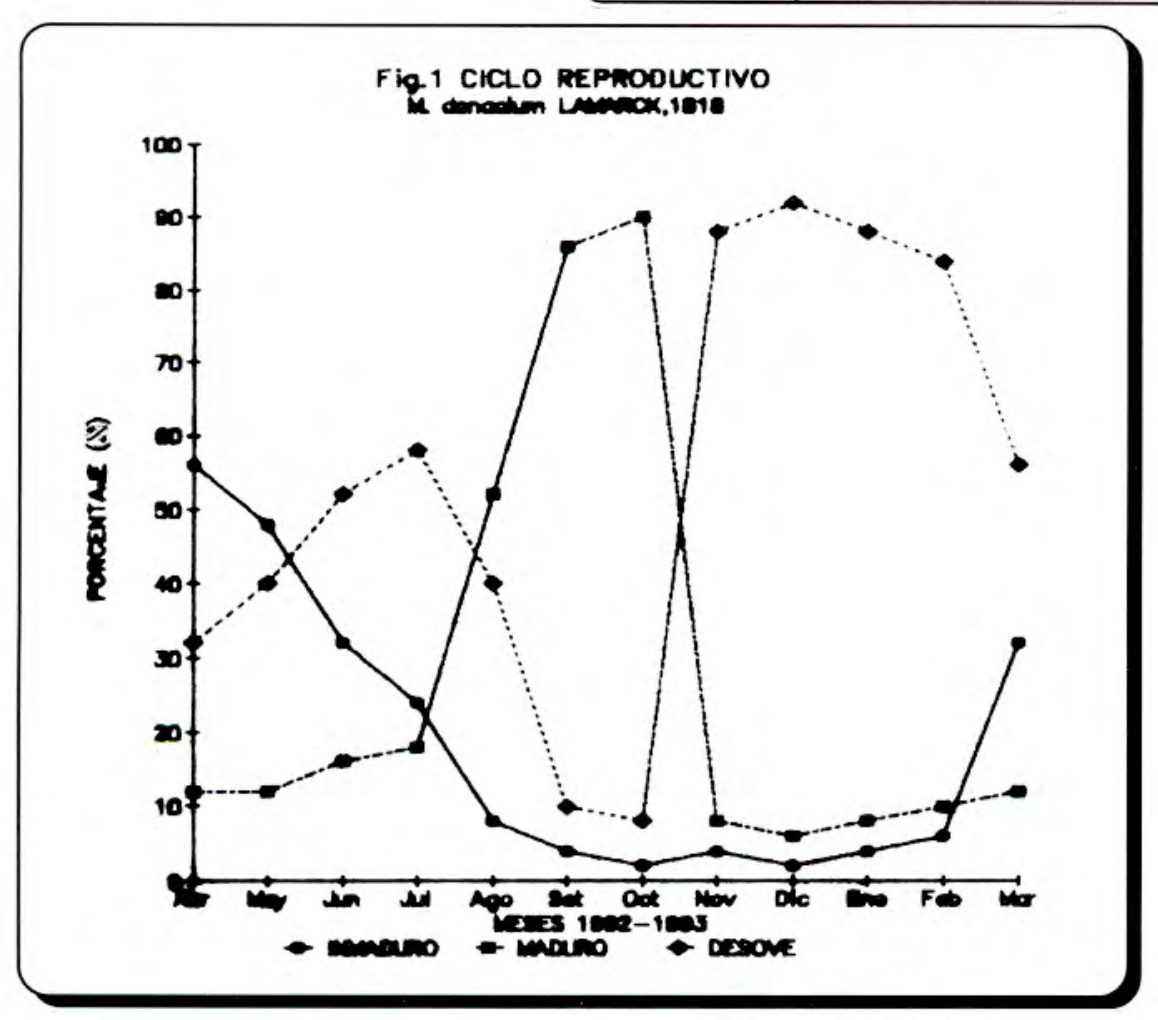

CICLO REPRODUCTIVO M. donacium LAMARCK, 1818 
Microfotografía A: ESTADO INMADURO
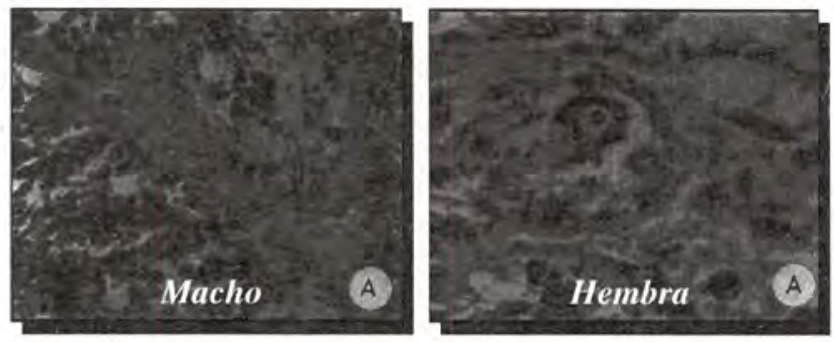

\subsubsection{Madúro}

Se observó que la mayor frecuencia de este estado se presentó entre los meses de agosto a octubre, con un pico máximo en el mes de octubre (90\%) (Tabla $\mathrm{N}^{\circ} 2$; Fig.1). Este estado representa el $27.5 \%$ de la muestra total considerada para el estudio reproductivo. Las microfotografías B muestran las características histológicas de este estado en individuos de los dos sexos.

\section{Microfotografía B: ESTADO MADURO}
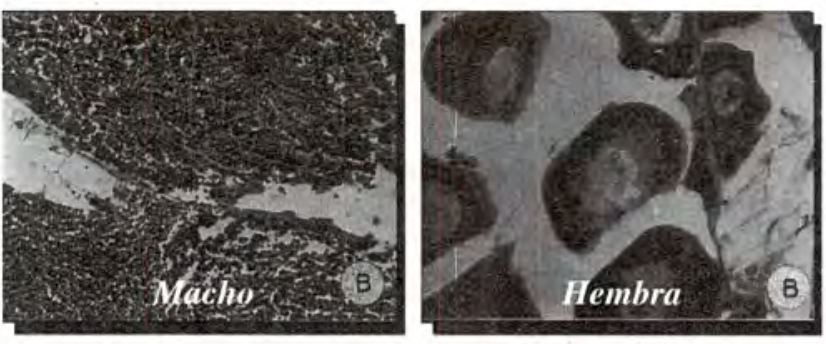

\subsubsection{Desove}

Comprendido entre los meses de noviembre y marzo con un pico máximo manifiesto en el mes de diciembre, representando este estado el $54 \%$ de la muestra total analizada, porque también presenta un desove menor entre los meses de abril a agosto, con un pico máximo en julio de 58\% (Tabla II; Fig.1). Las microfotografías C muestran las características histológicas de este estado tanto en machos como en hembras.

\section{Microfotografía C: ESTADO EN DESOVE}
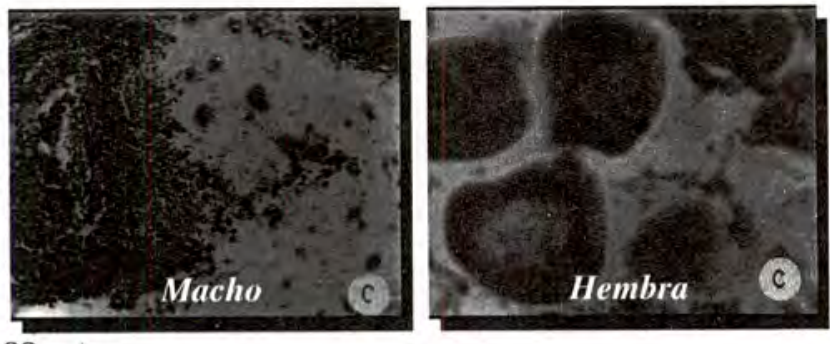

\section{DISCUSIÓN}

\subsection{PROPORCIÓN SEXUAL}

Se ha procedido a demostrar esta hipótesis por medio de $\mathrm{X}^{2}$ (ji-cuadrado) de la Tabla $\mathrm{N}^{\circ} 7$, indicando los resultados que existe una proporción de 1:1 generalizada para la macha. Si existe un grado de libertad para este test, y $\mathrm{X}^{2}$ es menor que 3.84 , considerado a un nivel de probabilidad del $95 \%$, determina que la hipótesis no sea rechazada del todo con la excepción de los meses de noviembre (1992) y enero (1993) que es algo mayor (5.12), por cuanto puede considerarse que la proporción sexual de la macha sea de 1:1 aún en la proporción anual.

\subsection{CICLO REPRODUCTIVO DE M. DONACIUM L.}

Los resultados obtenidos a través del periodo de muestreo, indican que presentan un ciclo sexual estacionable, abarcando todo el año (Fig. №1), con estados de desarrollo gonadales definidos y continuos; también con la existencia de un estado de reposo (reversión).

La Tabla $\mathrm{N}^{\circ} 2$ nos presenta 3 estados de desarrollo gonadal, apreciándose en la Fig. $\mathrm{N}^{\circ} 1$ una etapa de multiplicación y desarrollo, comprendida entre los meses de marzo a julio, con un pico elevado presente en el mes de abril (56\%). Otra etapa de maduración, manifiesta entre los meses de agosto a octubre, con un pico máximo entre los meses de setiembre y octubre (86 y 90\%) aparentemente; siguiendo la etapa de evacuación, que abarca los meses de noviembre a febrero; con un pico elevado en el mes de diciembre $(92 \%)$; aunque se evidencia que este proceso continúa todo el año con excepción de los meses de setiembre y octubre.

En resumen, los estados de desarrollo gonadal de la "macha" se establecenÿÿ de la siguiente manera: estado inmaduro: entre marzo y julio; estado maduro: entre agosto y octubre; estado de desove: entre noviembre y febrero.

\section{CONCLUSIONES}

La proporción sexual fue de 1:1 y la talla máxima encontrada fue de $92.5 \mathrm{~mm}$.

El ciclo reproductivo comprende tres estados: inmaduro (marzo-julio), maduro (agosto-octubre) y desove (todo el año, con mayor frecuencia en noviembre y febrero).

- La primera madurez sexual se manifiesta a los 60.0 $\mathrm{mm}$. de longitud total, siendo el promedio de individuos maduros de $77.2 \mathrm{~mm}$. 


\section{BIBLIOGRAFÍA}

ANGEL, CH. 1982. Maduración gonádica y fijación de Crassostrea rhizophorae en una laguna hipersalina del nororiente de Venezuela. Contribución № 48 . Est. de Inv. Mar. de Margarita. Fundación La Salle de Ciencias Naturales. pp. 215-239.

CHRISTIANSEN, H.E. 1981. Estructura de la dinámica de la población y biología de la almeja amarilla Mesodesma mactroides, Cap. II. Reproducción. Lust. Biol. Mar. Univ. Nac. de Buenos Aires. La Plata y del Sur. Comisiones de Investigaciones Científicas de la rovincia de Buenos Aires. Serie Contribuciones N2 27:3747.

DIFIORE, M.S. 1969. Diagnóstico Histológico. Tomo I. Sexta Edición. Edit. El Ateneo. Buenos Aires. Argentina. pp.4897.
CASTELLANOS, R. \& Col. 1980,81,82,83. : Evaluación de los recursos hidrobiológicos costeros (mariscos) Tacna-Moquegua. Vols. I-II-III-IV. Tacna,Perú.

LOPEZ, A. 1986. Etapas de desarrollo gonádico y determinación del ciclo reproductivo de Mesodesma donacium. Seminario de tesis para optar el título de profesor de estado en Biología y Ciencias. Universidad de Chile. Facultad de Ciencias. Dpto.de Biología. Sede Oriente.

SALGADO, L.I. y V. ISHIYAMA. 1979. Ciclo de madurez sexual y desove de macha Mesodesma donacium. Rev. Ciencias. Univ. Nac. Ciencias. Univ. Nac. Mayor de San Marcos 71 (1) :20-28.

TARIFEÑO, E. 1985. La macha (Mesodesma donacium, la marck, 1819) y sus posibilidades de cultivo. Inf. Mimeografiado, $33 \mathrm{p}$. 